\title{
Formation and Growth of Stacking Fault Tetrahedra in Ni via Vacancy Aggregation Mechanism
}

\author{
Dilpuneet S. Aidhy, ${ }^{1,2 *}$ Chenyang $\mathrm{Lu}^{3}, \mathrm{Ke} \mathrm{Jin}^{2}$, Hongbin $\mathrm{Bei}^{2}$, Yanwen Zhang ${ }^{2}$, Lumin \\ Wang $^{3}$, and William J. Weber ${ }^{4,2}$ \\ ${ }^{1}$ Department of Mechanical Engineering, University of Wyoming, Laramie, WY 82071, \\ USA \\ ${ }^{2}$ Materials Science and Technology Division, Oak Ridge National Laboratory, Oak \\ Ridge, TN 37831, USA \\ ${ }^{3}$ Department of Nuclear Engineering and Radiological Sciences, University of Michigan, \\ Ann Arbor, MI 48109, USA \\ ${ }^{4}$ Materials Science and Engineering, University of Tennessee, Knoxville, TN 37996 , \\ USA
}

This manuscript has been authored by UT-Battelle, LLC under Contract No. DE-AC05OOOR22725 with the U.S. Department of Energy. The United States Government retains and the publisher, by accepting the article for publication, acknowledges that the United States Government retains a non-exclusive, paid-up, irrevocable, world-wide license to publish or reproduce the published form of this manuscript, or allow others to do so, for United States Government purposes. The Department of Energy will provide public access to these results of federally sponsored research in accordance with the DOE Public Access Plan (http://energy.gov/downloads/doe-public-access-plan).

\section{*Email: daidhy@uwyo.edu}

Keywords

Molecular dynamics, diffusion, point defect, stacking fault tetrahedra

\begin{abstract}
Using molecular dynamics simulations, the formation and growth of stacking fault tetrahedra (SFT) are captured by vacancy cluster diffusion and aggregation mechanisms in Ni. The vacancytetrahedron acts as a nucleation point for SFT formation. Simulations show that perfect SFT can grow to the next size perfect SFT via a vacancy aggregation mechanism. The stopping and range of ions in matter (SRIM) calculations and transmission electron microscopy (TEM) observations reveal that SFT can form farther away from the initial cascade-event locations, indicating the operation of diffusion-based vacancy-aggregation mechanism.
\end{abstract}


Stacking-fault tetrahedra (SFT) are commonly observed defects in FCC metals during plastic deformation, melt-quench or irradiation conditions.[1-3] The most widely discussed SFT formation mechanism is by Silcox and Hirsh.[4] They proposed a dislocation glide based mechanism, according to which vacancies cluster to form dislocation loops bounded by Frank partials. These partials then dissociate into Shockley partials that glide towards the tetrahedron apex resulting in SFT formation. This mechanism has also been captured by molecular dynamics (MD) simulations in irradiated $\mathrm{Cu}$.[5] However, there remains a speculation about whether the nucleation of SFT (in melt-quench or irradiation) indeed requires condensation of vacancies into loops followed by their transformation into SFT, or if it could form by simple agglomeration of vacancies.[2, 6-10] De Jong and Koehler[2] initially postulated the vacancy-tetrahedron as a nucleation center for SFT, and later some experimental and theoretical studies indicated the possibility of this mechanism.[10-12] However, due to the lack of direct atomistic observation under experiments, and the absence of MD simulations that capture diffusion and clustering of vacancies to form SFT, the operation of such a mechanism has not yet been fully ascertained.[6, 7] In addition, while growth of SFT has been largely perceived to occur by accumulation of vacancies on SFT, and atomistic static calculations have been previously performed in this regard to understand the energy landscape and the vacancy-SFT interactions, $[13,14] \mathrm{MD}$ simulations have not yet dynamically captured the diffusion-based SFT growth to clearly show the operation of a vacancy aggregation mechanism. Furthermore, while previous studies have shown direct formation of SFT during a collision-cascade event, $[15,16]$ illustrating the SFT formation by the vacancy aggregation mechanism will indicate that they can also form much after the cascade event has occurred, or under non-cascade irradiation conditions (e.g., electrons, light ions and thermal neutrons) by the aggregation of irradiation-induced free vacancies.

In this work, using MD simulations, we demonstrate the formation of SFT via a vacancy agglomeration mechanism. Simulations reveal that a vacancy-tetrahedron cluster possibly acts as a nucleation center in the formation of SFT. While the individual vacancy has a high migration barrier, the vacancy clusters have significantly lower barriers that allow diffusion and clustering of small clusters to form large SFT. We also show that SFT growth can occur by a vacancy aggregation mechanism, and perfect SFT can grow to the next size perfect SFT configuration. The stopping and range of ions in matter (SRIM) prediction and our transmission electron microscope (TEM) observations show that SFT can form much deeper than the ion range where majority of the initial cascade-event occurs, supporting the operation of diffusion-based vacancy-aggregation mechanism.

To simulate SFT formation via this mechanism, we generate a random distribution of vacancies. The simulations are run at a high temperature of $1000 \mathrm{~K}$ to be able to capture vacancy diffusion on MD time scales. We model two FCC Ni systems, one using $20 \times 20 \times 20$ unit cells, and the other using $10 \times 10 \times 10$ unit cells consisting of 32,000 and 4,000 atoms respectively. The interatomic forces are calculated using the embedded atom method (EAM) based on the Bonny 2013 interatomic potential.[17] This potential is a modified version of the Bonny 2011 potential,[18] and predicts defect properties, such as migration and binding energies, in good agreement with $a b$ initio calculations.[17] The details of the potentials can be found elsewhere.[17] The 
simulations are carried out using the LAMMPS code.[19] Periodic boundary conditions are applied in all three directions, and a time step of $2 \mathrm{fs}$ ensures good energy conservation. The point defects are identified by comparing the perfect structure to the defected structure. During comparison with the perfect system, if a lattice site is unoccupied within a radial distance of 1 Angstrom, it is labeled as a vacancy. Similarly, if an original empty interstitial site is occupied, it is labeled as an interstitial.

Figure 1 shows the evolution of vacancies over time in an MD simulation. For this simulation, we use the larger system and create 100 random vacancies at the start of the simulation. The snapshots with projection of the cell in the $\langle 110\rangle$ direction are taken at $\mathrm{t}=0 \mathrm{~ns}, 8.2 \mathrm{~ns}, 22.8 \mathrm{~ns}$ and $83.8 \mathrm{~ns}$ intervals as shown in Figures 1 (a) - (d). A movie $\mathrm{S} 1$ at regular intervals is shown in the Supplemental section. Vacancies are represented in light blue, interstitials in pink, and the lattice atoms are in smaller size represented in dark blue. At the beginning of the simulation, all vacancies are individually present as shown in Figure 1a. With time, the vacancies start to cluster, as evidenced by the formation of two small clusters and a stacking fault tetrahedron in Figure $1 b$.

During vacancy clustering, new interstitials are self-created by the system; these interstitials, as discussed later, are created as a part of a building block of the SFT. Over time, these clusters begin to grow, and by $22.8 \mathrm{~ns}$ in Figure 1c, one of the smaller clusters has transformed into a large stacking fault tetrahedron. The system now has two SFT and one small cluster. Eventually, by $83.8 \mathrm{~ns}$ in Figure 1d, the leftover small cluster gets consumed by one of the stacking fault tetrahedron, and the two SFT further grow into large sizes by gradual vacancy accumulation. In the end, only a few individual vacancies are left behind as shown in Figure 1d. During this evolution, at different instances, various small vacancy clusters get created by vacancy aggregation; however, similar to the small cluster in Figure 1c, these clusters diffuse and join the two main SFT leading to their growth. Formation and diffusion of some of the small clusters is observable in the movie S1. In the end, no cluster-type other than a stacking fault tetrahedron remains stable. During the simulation, the two SFT also undergo significant rearrangement that requires them to change their shape and size as the individual vacancies and small clusters join them. By $83.8 \mathrm{~ns}$, the two SFT are of significantly large size; the one on the right is a 36 vacancy SFT. It is a perfect stacking fault tetrahedron containing the exact number of vacancies required for a stacking fault tetrahedron of magic number 36.[20] The other stacking fault tetrahedron contains 53 vacancies, which is 2 less than that of the next magic number stacking fault tetrahedron, i.e., 55. Eventually, this stacking fault tetrahedron will absorb two more vacancies from the leftover vacancies to form a perfect stacking fault tetrahedron. This simulation thus shows the formation of SFT by aggregation of individual vacancies without having the need to first form a dislocation loop. It is to be noted that we have not added interstitials in the system; thus, the simulation does not capture the mutual vacancy-interstitial recombination events that will likely slow down the kinetics of SFT formation. A schematic breakdown of SFT in a planar view is shown in Supplemental Section figure F1.

To understand the nucleation of SFT, Figure 2a shows a snapshot of the system at $1.8 \mathrm{~ns}$ from the simulation. By this time, while most of the vacancies are still present as isolated vacancies, some of the vacancies have clustered together to form four small clusters, as encircled. Two of the clusters are individually present, and the other two have 
diffused and joined each other. These clusters, named vacancy-tetrahedron (labeled $t 1$ in Figure 2b), consist of four vacancies as FCC nearest neighbors enclosing an interstitial atom inside them. It is formed when three vacancies aggregate together and prompt a fourth neighboring lattice atom to self-create a Frenkel pair,[21] thus forming a cluster of four vacancies and an interstitial. This is the cluster that was originally postulated by de Jong and Koehler[2] to be the nucleation site for SFT formation.

The energetics of formation of the vacancy-tetrahedron and ultimately a stacking fault tetrahedron containing 6 magic number vacancies is shown in Figure 2b. The energies of all the clusters are presented with reference to a structure containing 6 widely separated randomly distributed vacancies in a $10 \times 10 \times 10$ unit cells system. A cluster, e.g., a di-vacancy cluster (labeled di) is formed by clustering two vacancies together and leaving the remaining four vacancies at the same locations as that in the reference structure. As expected, there is a positive binding energy when a di-vacancy cluster is formed, and it increases as the cluster size increases from di to tri to tetrahedron ( $\mathrm{t} 1)$, showing that the system prefers self-creation of a Frenkel pair in a $\mathrm{t} 1$ cluster. The next cluster is labeled $\mathrm{t} 1+\mathrm{di}$, which is formed when two more vacancies join the tetrahedron in the $\langle 110\rangle$ direction; the binding energy further increases when the second tetrahedron is completed by creation of another Frenkel pair as shown in $t 1+t 2$ cluster. The sixth (final) vacancy joins the cluster as shown in $t 1+t 2+1$ vac configuration. Beyond this configuration, the third and fourth tetrahedra are then completed by self-creation of two more Frenkel pairs as shown in $\mathrm{t} 1+\mathrm{t} 2+\mathrm{t} 3$ and $\mathrm{t} 1+\mathrm{t} 2+\mathrm{t} 3+\mathrm{t} 4$, thus completing a perfect sixvacancy stacking fault tetrahedron.

The vacancy-tetrahedron largely acts as a nucleation point for the formation of a stacking fault tetrahedron. Not only is it more stable than a di-vacancy by $0.41 \mathrm{eV}$, its binding energy with other smaller clusters is also much higher leading to formation of SFT. This can be fairly understood by a comparison between a di-vacancy and t1 interaction with another di-vacancy as shown in Figure 2c. The difference between the two is $0.85 \mathrm{eV}$ illustrating that when a diffusing di-vacancy comes in the vicinity of vacancy-tetrahedron, it would bind much more strongly with it than compared to a divacancy.

Now we show SFT growth via vacancy aggregation mechanism. Figure 3a shows a 21-vacancy perfect stacking fault tetrahedron projected in $\langle 111\rangle$ direction. Crystallographically, a 21-vacancy stacking fault tetrahedron has 6 vacancies on its edge; the edge-vacancies are shown in the inset more clearly. 10 randomly distributed vacancies are added to the system as shown in Figure 3a to capture the aggregation mechanism, and to investigate if a perfect stacking fault tetrahedron (21-magic number) could grow to the next perfect stacking fault tetrahedron (i.e., 28-magic number). Eight vacancies are visible while the other two are behind the SFT. The simulation is run for $66.4 \mathrm{~ns}$, and the snapshots are shown at $18 \mathrm{~ns}, 53.8 \mathrm{~ns}$ and $66.4 \mathrm{~ns}$ in Figures 3b-3d respectively. A movie S2 showing the SFT growth at regular intervals is given in the Supplemental section. Four vacancies join the SFT by $18 \mathrm{~ns}$, six by $53.5 \mathrm{~ns}$ and seven by $66.4 \mathrm{~ns}$ thus growing and completing a perfect stacking fault tetrahedron. The three extra vacancies remain individually present in the system. During the growth, we often find that an interacting vacancy creates metastable vacancy-tetrahedron on SFT faces before it finds a stable site. Such atomic rearrangements on SFT faces can be observed in the 
movie. The stacking fault tetrahedron however maintains its overall structure and initial location during the growth process. SFT growth by vacancy accumulation has been previously studied in $\mathrm{Cu}$ and $\mathrm{Al}$ using static simulations.[13, 14] Those simulations showed that albeit the preferred sites for vacancy accumulation could be different for the two materials (i.e., either at the edge or corner of the tetrahedron), there is strong preference for vacancy binding on SFT faces. Thus, in agreement with previous studies, this simulation captures the dynamical SFT growth by vacancy accumulation.

Diffusion plays an important role in the formation and growth of SFT. It is quite evident from various studies that clustering of vacancies significantly decreases migration barriers. Previous work by Lam et al [22] on FCC Cu showed that the defect migration barrier decreased from $0.82 \mathrm{eV}$ to $0.55 \mathrm{eV}$ from mono to di-vacancy. Similarly, Sabochick and Yip[23] showed that the barriers further decrease to $0.56 \mathrm{eV}$ and $0.38 \mathrm{eV}$ in tri and tetravacancy clusters. Our nudged elastic band (NEB) calculations on first nearest neighbor vacancy diffusion in $\mathrm{Ni}$ also show similar trends where migration barriers decrease from $1.09 \mathrm{eV}$ to $0.5 \mathrm{eV}$ from mono to tri-vacancies. Low migration barriers coupled with high binding energies enhance possibilities of SFT formation. It is important to mention that Martínez and Uberuaga[24] recently proposed another diffusion-based SFT growth mechanism. They showed that small imperfect SFT have high diffusivities that allow them to mutually interact and grow to form perfect SFT. Thus, in view of the above discussion, fast diffusion of defects promotes growth of both imperfect and perfect SFT.

Diffusion-based SFT formation indicates that, under irradiation conditions, SFT may not necessarily form only during cascade events. Previous MD simulations have captured SFT formed directly due to displacement cascades.[15, 16] Other simulations have considered the presence of a void or vacancy rich regions as the nucleation point for the formation of SFT.[5, 25, 26] While these simulations have unraveled different SFT formation mechanisms, they have not focused on the isolated irradiation-induced free vacancies. SFT formation by mere diffusion of vacancies and small vacancy cluster now shows that they could form much farther away from the collision cascade. This prediction finds ground from the SRIM prediction and our TEM observation that show formation of SFT much farther away from the cascade event. Figure 4 (a) and (b) shows the comparison of the damage-depth profile in pure $\mathrm{Ni}$ irradiated with $3.0 \mathrm{MeV} \mathrm{Au}^{2+}$ to the fluence of $5 \times 10^{15} \mathrm{~cm}^{-2}$, between the prediction from SRIM (in displacements per atom) and the TEM characterization. The SRIM prediction is based on the quick calculation of damage assuming $\mathrm{Ni}$ density of $8.908 \mathrm{~g} \mathrm{~cm}^{-3}$ with displacement energy of $40 \mathrm{eV}$. As shown in Fig. 4(a), the Au ion induced damage peaks at $\sim 155 \mathrm{~nm}$ with most of Ni atoms within the first $250 \mathrm{~nm}$ displaced in average more than 20 times, and the implanted $\mathrm{Au}$ ion stops within the first half micrometer with peak concentration less than $0.3 \%$. Figure 4 (b) shows the bright-filed TEM image for the same sample in the same depth scale. We find that TEM-revealed damage range is much deeper than the SRIM prediction, indicating significant migration of defects. It is known that some channeled Au ions may penetrate deeper than the SRIM prediction, the possibility is low as the Au irradiations were performed a few degrees off the surface normal, in the $\langle 100\rangle$ direction. At the depth where, according to the SRIM prediction, most displacement events occur, the defect density is relatively low; however, at deeper region (>700 nm in Fig. 4(b)) where 
the ions rarely reach, high dense dislocations are observed. Figure 4 (c) is a TEM image highlighting various SFT in the damaged region in Figure 4 (b) as shown by the square and the arrow. These experimental observations thus reveal that SFT can form much deeper (or farther) away from the initial region of ion damage.

In conclusion, MD simulations show that SFT formation could indeed occur by mere aggregation of vacancies, and vacancy-tetrahedron could possibly act as a nucleation agent, as initially proposed by de Jung and Koehler. The initial condensation of vacancies into dislocation loops is not necessary. The simulations also capture the growth of perfect SFT by vacancy aggregation mechanism. Finally, SRIM and TEM observations show that SFT can form much farther away from the initial cascade-event locations, indicating the operation of diffusion-based vacancy-aggregation mechanism.

Acknowledgement: This work was supported by Energy Dissipation to Defect Evolution (EDDE), an Energy Frontier Research Center funded by the U.S. Department of Energy, Office of Science, Basic Energy Sciences. The computer simulations were performed at the National Energy Research Scientific Computing Center at Lawrence Berkeley National Laboratory, which is supported by the Office of Science, U.S. Department of Energy under Contract No. DEAC02-05CH11231.

\section{References}

[1] M. Kiritani, Materials Chemistry and Physics, 50 (1997) 133-138.

[2] M. de Jong, J.S. Koehler, Physical Review, 129 (1963) 49-61.

[3] B.N. Singh, S.J. Zinkle, Journal of Nuclear Materials, 206 (1993) 221-229.

[4] J. Silcox, P.B. Hirsch, Philosophical Magazine, 4 (1959) 72-89.

[5] B.D. Wirth, V. Bulatov, T.D.d.1. Rubia, Journal of Nuclear Materials, 283-287 (2000) 773-777.

[6] M.H. Loretto, P.J. Phillips, M.J. Mills, Scripta Mater, 94 (2015) 1-4.

[7] B.N. Singh, S.I. Golubov, H. Trinkaus, D.J. Edwards, M. Eldrup, Journal of Nuclear Materials, 328 (2004) 77-87.

[8] R. Schibli, R. Schäublin, Journal of Nuclear Materials, 442 (2013) S761-S767.

[9] H. Kimura, D. Kuhlmann-Wilsdorf, R. Maddin, Applied Physics Letters, 3 (1963) 45.

[10] Y. Shirai, K. Furukawa, J. Takamura, W. Yamada, S. Iwata, Applied Physics A, 37 (1985) 65-72.

[11] M. Doyama, R.M.J. Cotterill, Physical Review, 137 (1965) A994-A999.

[12] R.M.J. Cotterill, Philosophical Magazine, 6 (1961) 1351-1362.

[13] Y.N. Osetsky, A. Serra, M. Victoria, S.I. Golubov, V. Priego, Philosphical Magazine A, 79 (1999) 2285-2311.

[14] H. Wang, D.S. Xu, R. Yang, P. Veyssière, Acta Materialia, 59 (2011) 10-18.

[15] Y.N. Osetsky, D.J. Bacon, Nuclear Instruments \& Methods in Physics Research Section B, 180 (2001) 85-90. 
[16] K. Nordlund, F. Gao, Applied Physics Letters, 74 (1999) 2720-2722.

[17] G. Bonny, N. Castin, D. Terentyev, Modelling and Simulation in Materials Science and Engineering, 21 (2013) 085004.

[18] G. Bonny, D. Terentyev, R.C. Pasianot, S. Poncé, A. Bakaev, Modelling and Simulation in Materials Science and Engineering, 19 (2011) 085008.

[19] S. Plimpton, Journal of Computational Physics, 117 (1995) 1-19.

[20] (The magic number of SFTs are 3, 6, 10, 15, 21, 28, 36, 55 ...).

[21] (Although Frenkel pairs are referred to defects which are stable and substantially far apart, here the term is used for simplicity.).

[22] N.Q. Lam, N.V. Doan, L. Dagens, J. Phys. F: Met. Phys., 15 (1985) 799-808.

[23] M.J. Sabochick, S. Yip, J Phys. F. Met. Phys, 18 (1988) 1688-1701.

[24] E. Martinez, B.P. Uberuaga, Sci Rep, 5 (2015) 9084.

[25] B. Uberuaga, R. Hoagland, A. Voter, S. Valone, Physical Review Letters, 99 (2007).

[26] H. Wang, D.S. Xu, R. Yang, P. Veyssière, Acta Materialia, 59 (2011) 1-9. 


\section{Figures}
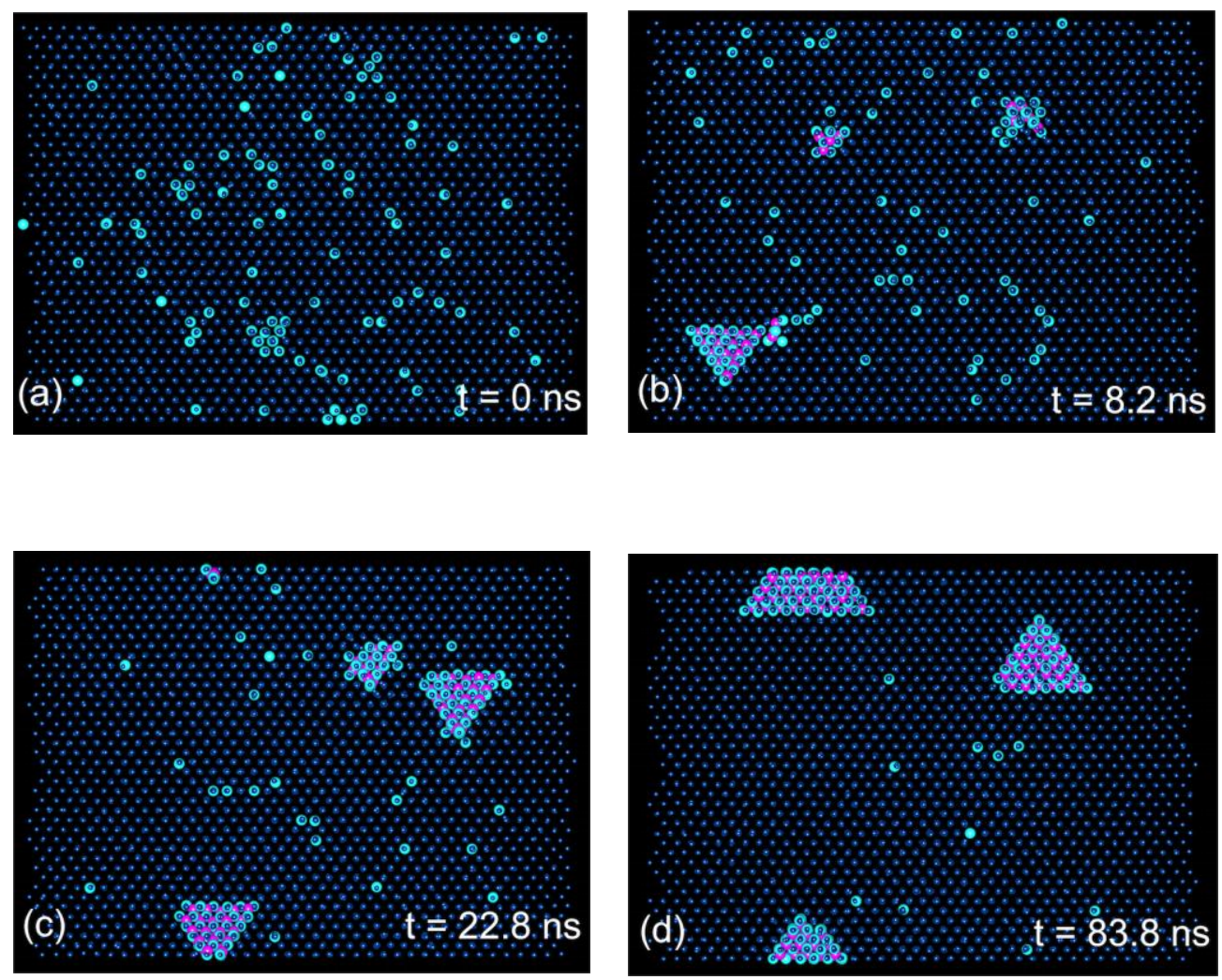

Figure 1. Vacancy evolution during MD simulation leading to formation of two SFT. Snapshots are taken with the cell projected in $\langle 110\rangle$ direction. 

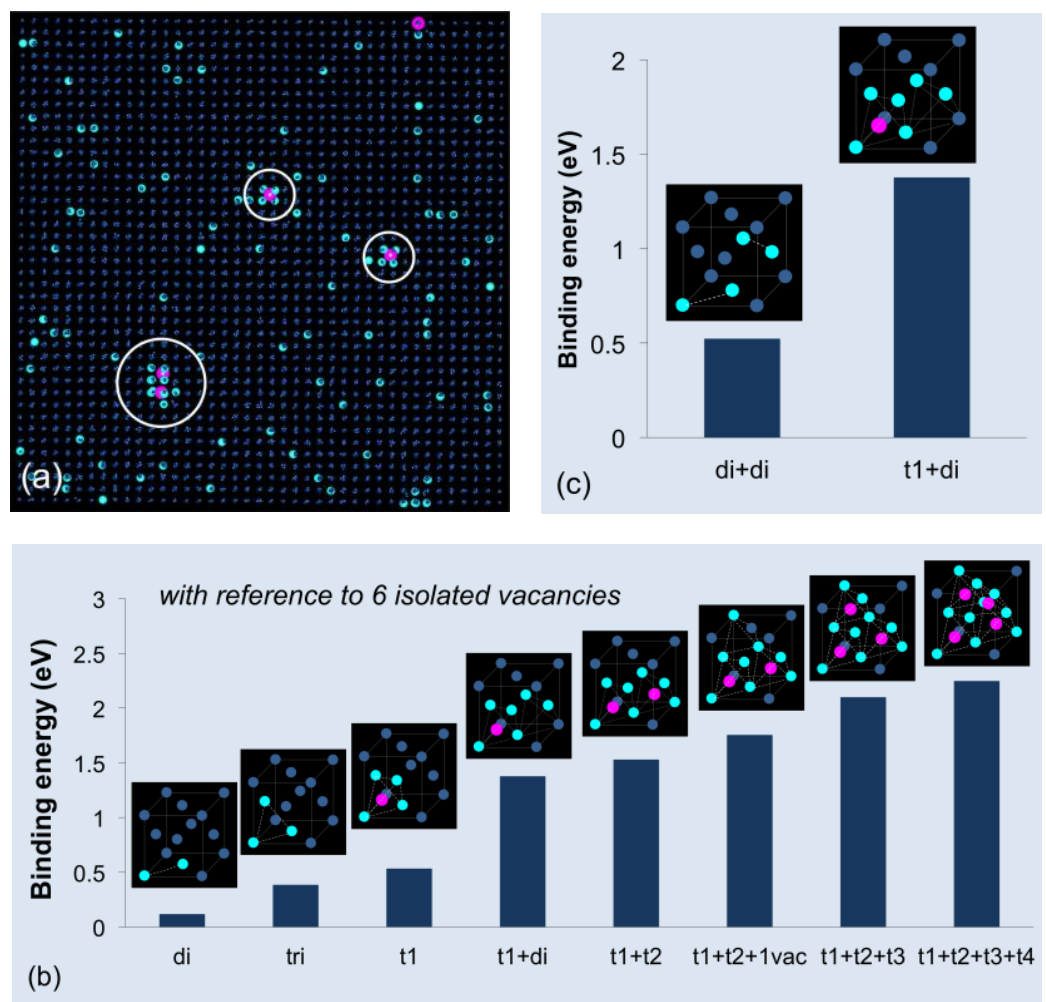

Figure 2. (a) Snapshot of the simulation cell from figure 1 at 1.8 ns. (b) Binding energies of various clusters with reference to 6 isolated vacancies. (c) Comparison of binding energies between di and tetrahedron with di-vacancies. 

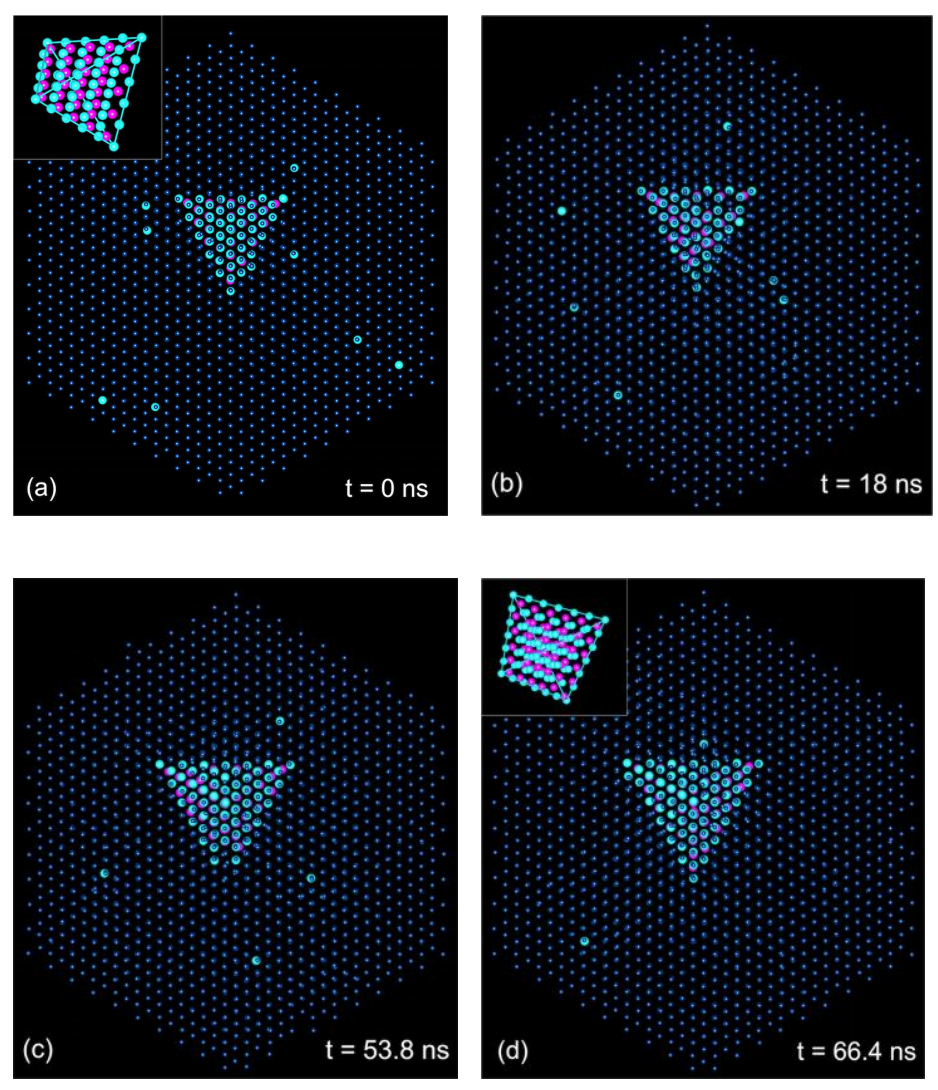

Figure 3. Growth of a perfect stacking fault tetrahedron containing 21-magic number vacancies to a 28-magic number vacancies' stacking fault tetrahedron by vacancy aggregation. Snapshots taken with projection of the cell in $\langle 111\rangle$ direction. (a) A 21magic number stacking fault tetrahedron surrounded by 10 randomly distributed vacancies at $\mathrm{t}=0 \mathrm{~ns}$. Four vacancies join in (b), six in (c) and seven in (d) at $18 \mathrm{~ns}, 53.8$ ns and 66.4 ns respectively. A close view of stacking fault tetrahedron are shown in the inset in (a) and (d). 

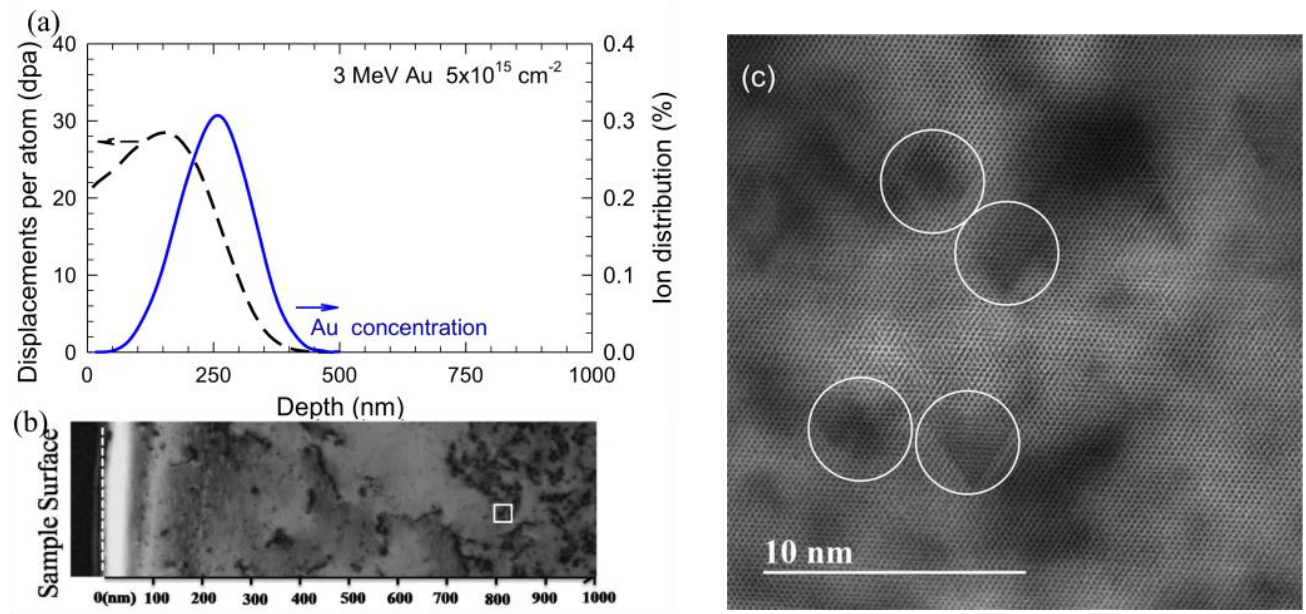

Figure 4. (a) Displacement profile (left axis) and Au distribution (right axis) predicted by SRIM of pure Ni irradiated with $3.0 \mathrm{MeV}$ Au ions to the fluence of $5 \times 10^{15} \mathrm{~cm}^{-2}$. (c) Various SFT highlighted by circles observed under TEM in the highlighted sample region as shown in (b). 
Graphical abstract

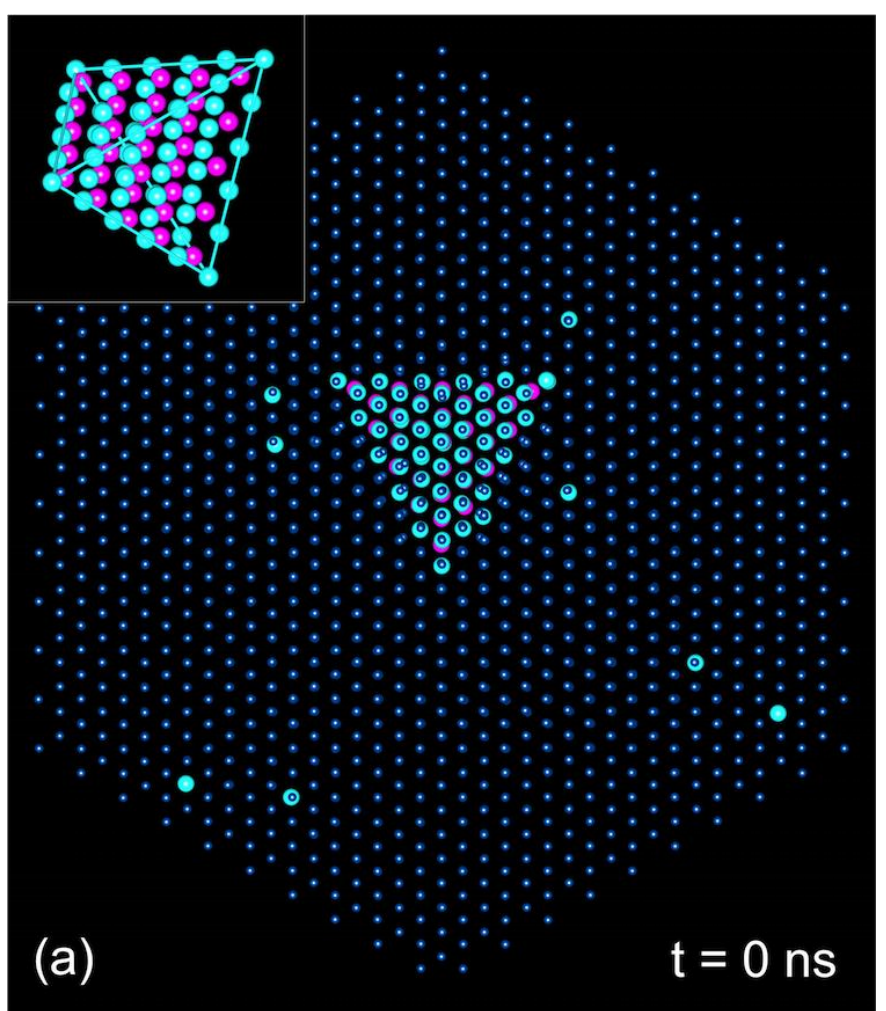

